\title{
No-go results for non-topological solitons in some types of gauge field theories
}

\section{Mikhail N. Smolyakov*}

Skobeltsyn Institute of Nuclear Physics, Moscow State University, Moscow, 119991, Russia E-mail: smolyakovetheory.sinp.msu.ru

The conditions, under which non-topological solitons periodic in time are absent in the theory describing the Yang-Mills field coupled to a scalar field, are derived. It is also shown that nontopological solitons of such type are absent in the theory describing the U(1)-charged massive vector field.

The XXth International Workshop High Energy Physics and Quantum Field Theory September 24-October 1, 2011

Sochi Russia

\footnotetext{
* Speaker.
} 


\section{Introduction}

The well-known Derrick theorem (sometimes referred to as the Hobart-Derrick theorem) [प, [] states that there are no static solitons (i.e. static solutions to the corresponding equations of motion, possessing finite energy localized in a finite domain of space) in the (3+1)-dimensional scalar field theory with the action $(\mu=0,1,2,3)$

$$
S=\int d^{4} x\left(\frac{1}{2} \partial^{\mu} \phi \partial_{\mu} \phi-V(\phi)\right)
$$

provided

$$
V(\phi) \geq 0
$$

The proof is very simple. Indeed, let us suppose that there exists a soliton solution $\phi(\vec{x})$, we also suppose that there exists vacuum solution $\phi=\phi_{0}$ such that $V\left(\phi_{0}\right)=0$ (if $V\left(\phi_{0}\right) \neq 0$, we can always take the potential $\tilde{V}(\phi)=V(\phi)-V\left(\phi_{0}\right)$ instead of the initial one), where $\phi_{0}$ is a constant. Now let us consider the following transformation of our solution

$$
\phi(\vec{x}) \rightarrow \phi(\lambda \vec{x})
$$

with a real parameter $\lambda$. The action on this transformed solution takes the form (we can drop the integration by $t$ in the action because our solution is supposed to be static)

$$
S=-\lambda^{-1} \frac{1}{2} \int d^{3} x \partial^{i} \phi \partial_{i} \phi-\lambda^{-3} \int d^{3} x V(\phi(\vec{x})),
$$

where $i=1,2,3$. Since we suppose that $\phi(\vec{x})$ is a solution to the equation of motion, the variation of the action on this solution should vanish for any variations of the field. It means that

$$
\left.\frac{d S}{d \lambda}\right|_{\lambda=1}=\frac{1}{2} \int d^{3} x \partial^{i} \phi \partial_{i} \phi+3 \int d^{3} x V(\phi(\vec{x}))=0 .
$$

Indeed, $\lambda=1$ in $([\mathbb{L} .3)$ corresponds to the solution. We see, that if $V(\phi) \geq 0$, then the only solution

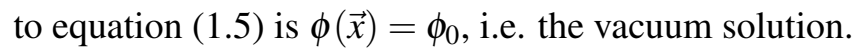

Later analogous scaling methods were applied to more complicated static configurations of fields, for example, to the Yang-Mills field coupled to a scalar field [B]], to skyrmions [四], monopoles [四] and instantons [四, [1, 目].

Apart from taking potentials which are not non-negative, there is another simple way to overcome the restriction put by the Derrick theorem. Indeed, one can consider a time-dependent solution for the scalar field, for example, of the form

$$
\phi(t, \vec{x})=e^{i \omega t} \varphi(\vec{x})
$$

Nevertheless, it is possible to obtain restrictions, analogous to the one of the Derrick theorem, for time-dependent solutions also. This can be done by utilizing slightly modified form of transformation $(\mathbb{L} \cdot 3]$ ) (such transformations were applied in $\left[[\mathbb{Z}, \mathbb{Z}]\right.$ to models with $\sim e^{i \omega t}$ time dependence of fields and in [ [ ] to a model describing single scalar field periodic in time with a period $T$ ). We will discuss this possibility and the corresponding transformations in an explicit form in the next sections. 


\section{Yang-Mills field coupled to a scalar field}

Let us take the following form of the four-dimensional action:

$$
S=\int d^{4} x\left[\eta^{\mu v}\left(D_{\mu} \phi\right)^{\dagger} D_{v} \phi-V\left(\phi^{\dagger} \phi\right)-\frac{1}{4} F^{a \mu v} F_{\mu v}^{a}\right],
$$

where $\eta_{\mu v}=\operatorname{diag}(1,-1,-1,-1)$ is the flat Minkowski metric,

$$
\begin{gathered}
F_{\mu \nu}^{a}=\partial_{\mu} A_{v}^{a}-\partial_{\nu} A_{\mu}^{a}+g C^{a b c} A_{\mu}^{b} A_{v}^{c}, \\
D_{\mu} \phi=\partial_{\mu} \phi-i g T^{a} A_{\mu}^{a} \phi,
\end{gathered}
$$

where $C_{a b c}$ are the structure constants of a compact gauge group and $T^{a}$ are generators of the group in the representation space of field $\phi$. We also suppose that

$$
\left.V\left(\phi^{\dagger} \phi\right)\right|_{\phi^{\dagger} \phi=0}=0, \quad\left|\frac{d V\left(\phi^{\dagger} \phi\right)}{d\left(\phi^{\dagger} \phi\right)}\right|_{\phi^{\dagger} \phi=0}<\infty
$$

(the latter condition ensures that the vacuum solution is $\phi \equiv 0, A_{\mu}=g T^{a} A_{\mu}^{a} \equiv 0$ ) and:

1. there are no sources which are external to the system described by action ([2. 1$)$;

2. solutions to equations of motion are periodic in time with a period $T<\infty$, i.e. for all fields on the solution the relation $\Psi(t+T, \vec{x}) \equiv \Psi(t, \vec{x})$ must hold for any $t$, where $\Psi(t, \vec{x})$ schematically represents the field under consideration (more general periodicity conditions, leading to the same results as those which will be obtained below, can be found in [ $[\mathrm{Q}]$ ).

Using the periodicity conditions, we can rewrite the initial action as

$$
\begin{gathered}
S=\int_{-\infty}^{\infty} d t \int d^{3} x L[\Psi(t, \vec{x})]=\sum_{n=-\infty}^{\infty} \int_{n T}^{(n+1) T} d t \int d^{3} x L[\Psi(t, \vec{x})]= \\
=\sum_{n=-\infty}^{\infty} \int_{0}^{T} d t \int d^{3} x L[\Psi(t+n T, \vec{x})]=\sum_{n=-\infty}^{\infty} \int_{0}^{T} d t \int d^{3} x L[\Psi(t, \vec{x})] .
\end{gathered}
$$

Thus, we can use the effective action

$$
S_{\text {eff }}=\int_{0}^{T} d t \int d^{3} x L[\Psi(t, \vec{x})]
$$

instead of the initial one. The necessity of our restriction to consider solutions periodic in time is due to the fact that the method of obtaining no-go results, which will be used below, can not be applied to infinite integrals; for periodic solutions we can take the effective action of form ([2.6), which is finite by construction.

We will be looking for smooth solutions to equations of motion, following from (ㅁ. that all the fields tend to their vacuum values at $x^{i} \rightarrow \pm \infty$. Let us denote

$$
\begin{aligned}
\int_{0}^{T} d t \int d^{3} x\left(D_{0} \phi\right)^{\dagger} D_{0} \phi & =\Pi_{0} \geq 0 \\
\int_{0}^{T} d t \int d^{3} x\left(D_{i} \phi\right)^{\dagger} D_{i} \phi & =\Pi_{1} \geq 0 \\
\int_{0}^{T} d t \int d^{3} x \frac{1}{2} F_{0 i}^{a} F_{0 i}^{a} & =\Pi_{A 0} \geq 0 \\
\int_{0}^{T} d t \int d^{3} x \frac{1}{4} F_{i j}^{a} F_{i j}^{a} & =\Pi_{A 1} \geq 0
\end{aligned}
$$


where $i, j=1,2,3$. All these integrals are supposed to be finite. The corresponding effective action in this case takes the form

$$
S=\Pi_{0}-\Pi_{1}-\int_{0}^{T} d t \int d^{3} x V\left(\phi^{\dagger} \phi\right)+\Pi_{A 0}-\Pi_{A 1}
$$

Now let us consider the following transformation of our solution

$$
\begin{array}{r}
\phi(t, \vec{x}) \rightarrow \lambda^{\gamma} \phi(t, \lambda \vec{x}), \\
A_{0}^{a}(t, \vec{x}) \rightarrow A_{0}^{a}(t, \lambda \vec{x}), \\
A_{i}^{a}(t, \vec{x}) \rightarrow \lambda A_{i}^{a}(t, \lambda \vec{x})
\end{array}
$$

with a real parameter $\lambda$. Repeating the steps presented in the Introduction, from the equality $\left.\frac{d S}{d \lambda}\right|_{\lambda=1}=0$ we get

$$
\begin{array}{r}
(2 \gamma-3) \Pi_{0}-(2 \gamma-1) \Pi_{1}- \\
-\int_{0}^{T} d t \int d^{3} x\left(2 \gamma \frac{d V\left(\phi^{\dagger} \phi\right)}{d\left(\phi^{\dagger} \phi\right)} \phi^{\dagger} \phi-3 V\left(\phi^{\dagger} \phi\right)\right)-\Pi_{A 0}-\Pi_{A 1}=0 .
\end{array}
$$

It is not difficult to show (see [Q] for detailed calculations), that if integrals $\int_{0}^{T} d t \int d^{3} x \frac{d V\left(\phi^{\dagger} \phi\right)}{d\left(\phi^{\dagger} \phi\right)} \phi^{\dagger} \phi$, $\int_{0}^{T} d t \int d^{3} x V\left(\phi^{\dagger} \phi\right)$ are finite and if there exists $\gamma: \frac{1}{2}<\gamma \leq \frac{3}{2}$ for which the inequality

$$
2 \gamma \frac{d V\left(\phi^{\dagger} \phi\right)}{d\left(\phi^{\dagger} \phi\right)} \phi^{\dagger} \phi-3 V\left(\phi^{\dagger} \phi\right) \geq 0
$$

is fulfilled for any $\phi$, then ([.J5) leads to $\phi \equiv 0$ and $F_{\mu \nu}^{a} \equiv 0$ (the latter equality means that $A_{\mu}$ is a pure gauge and we can set $\left.A_{\mu} \equiv 0\right)$. It means, that under these restrictions non-topological solitons with the fields vanishing at spatial infinity are absent in the theory with action (2.1).

It is evident, that the restriction presented above is also valid for the models with the scalar field only, i.e. if we drop the gauge field from the theory; and in the pure Yang-Mills theory (the absence of non-topological solitons in the pure Yang-Mills theory was shown long time ago for the static [ए]], periodic [ए]] and general cases [ए2], the corresponding conjecture was discussed earlier, see, for example, [[1]] and references therein).

Eq. (2.16) with $\gamma=3 / 2$ results in

$$
\frac{d V\left(\phi^{\dagger} \phi\right)}{d\left(\phi^{\dagger} \phi\right)} \phi^{\dagger} \phi-V\left(\phi^{\dagger} \phi\right) \geq 0
$$

For the first time the no-go condition ([D] $)$ for $V\left(\phi^{\dagger} \phi\right) \geq 0$ and the potentials $V\left(\phi^{\dagger} \phi\right)$ including a positive mass term was obtained in a different way in [IU]] (for the case of a single complex scalar field coupled to electromagnetic field this restriction was obtained in [15]]).

It is necessary to note that sometimes the known restriction ([2.17) is not sufficient to provide an information about non-existence of non-topological solitons. Indeed, as an example let us take a potential of the form $V\left(\phi^{\dagger} \phi\right)=q_{1}\left(\phi^{\dagger} \phi\right)^{4}-q_{2}\left(\phi^{\dagger} \phi\right)^{2}$ with $q_{1}>0, q_{2}>0$ (note, that we can consider $\phi \equiv 0$ as the "vacuum" from the mathematical point of view, but it is the local maximum of the scalar field potential and thus such a configuration is unstable and can not be considered as 
the vacuum from the physical point of view). For this potential equality (․ㅣ) does not hold for any $\phi$. Meanwhile, substituting the potential into (R.16) and taking $\gamma=\frac{3}{4}$ we get $q_{1}\left(\phi^{\dagger} \phi\right)^{4} \geq 0$, which means that there are no solutions corresponding to a non-topological soliton in such a theory. Thus, in some cases general restriction (R.T6) can be very useful.

One more remark is in order. The surface terms at $t=0$ and $t=T$, which arise when obtaining equations of motion from the effective action (R.DI) with the help of variations of fields following from (2.12)-(2.14), appear to be modulo equal and cancel each other. This ensures that the equations of motion obtained from the initial action and those obtained from the effective action coincide, as well as possible solutions, and the procedure of obtaining the no-go results appears to be consistent with the equations of motion coming from the original action (problems, which can arise due to non-zero surface terms in the case of the Derrick theorem applied to a finite spacetime domain, were discussed in [ए]]). The same is valid, of course, for the model which will be discussed in the next section.

\section{U(1)-charged massive vector field}

As a second example let us consider a massive complex vector field in the $\mathrm{U}(1)$ gauge theory. The corresponding action has the form

$$
S=\int d^{4} x\left[-\frac{1}{2} \eta^{\mu \rho} \eta^{v \sigma} W_{\mu \nu}^{-} W_{\rho \sigma}^{+}+m^{2} \eta^{\mu v} W_{\mu}^{-} W_{v}^{+}-\frac{1}{4} F^{\mu v} F_{\mu v}\right]
$$

where $m \neq 0$ and

$$
\begin{gathered}
F_{\mu v}=\partial_{\mu} A_{v}-\partial_{v} A_{\mu}, \\
D_{\mu} W_{v}^{ \pm}=\partial_{\mu} W_{v}^{ \pm} \mp i e A_{\mu} W_{v}^{ \pm}, \\
W_{\mu v}^{ \pm}=D_{\mu} W_{v}^{ \pm}-D_{v} W_{\mu}^{ \pm} .
\end{gathered}
$$

We also suppose that there are no sources which are external to this system; all fields are smooth and vanish at spatial infinity; solutions to equations of motion are periodic in time with a period $T$. As in the case of the Yang-Mills field coupled to a scalar field, we can use the effective action where integration in $t$ is performed in the limits $[0, T]$. Of course, we suppose that all integrals in this effective action are finite.

Analogously to what was made in the previous section, we consider the following transformation of a supposed solution:

$$
\begin{array}{r}
W_{0}^{ \pm}(t, \vec{x}) \rightarrow \lambda^{\frac{1}{2}} W_{0}^{ \pm}(t, \lambda \vec{x}), \\
W_{i}^{ \pm}(t, \vec{x}) \rightarrow \lambda^{\frac{3}{2}} W_{i}^{ \pm}(t, \lambda \vec{x}), \\
A_{0}^{a}(t, \vec{x}) \rightarrow A_{0}^{a}(t, \lambda \vec{x}), \\
A_{i}^{a}(t, \vec{x}) \rightarrow \lambda A_{i}^{a}(t, \lambda \vec{x})
\end{array}
$$

with a real parameter $\lambda$. It is not difficult to show (just repeating the steps presented in the previous section, see also [Q] for details) that the use of these transformations leads to $A_{\mu} \equiv 0, W_{0}^{ \pm} \equiv 0$ and $W_{i j}^{ \pm} \equiv 0$. With $A_{\mu} \equiv 0$ equality $W_{i j}^{ \pm} \equiv 0$ can be rewritten as

$$
\partial_{i} W_{j}^{ \pm}-\partial_{j} W_{i}^{ \pm} \equiv 0
$$


Now let us take the equations of motion for the field $W_{\mu}^{ \pm}$with $A_{\mu} \equiv 0$. It follows from these equations (taking into account $W_{0}^{ \pm} \equiv 0$ ) that

$$
\partial^{i} W_{i}^{ \pm}=0
$$

Equations (B.9) and (B.TO) imply that

$$
\begin{array}{r}
W_{i}^{ \pm}=\partial_{i} \varphi^{ \pm}, \\
\Delta \varphi^{ \pm}=0,
\end{array}
$$

where $\varphi^{ \pm}=\varphi^{ \pm}(t, \vec{x}),\left(\varphi^{+}\right)^{*}=\varphi^{-}$. The condition $\int d^{3} x W_{i}^{-} W_{i}^{+}=\int d^{3} x \partial_{i} \varphi^{-} \partial_{i} \varphi^{+}<\infty$ (which follows from the demand to have finite integrals in the action) clearly leads to $\varphi^{ \pm}=\varphi^{ \pm}(t)$ (to show it one can simply use the results of the Derrick theorem discussed in the Introduction) and thus

$$
W_{i}^{ \pm} \equiv 0
$$

leading to

$$
W_{\mu}^{ \pm} \equiv 0
$$

The latter equality together with $A_{\mu} \equiv 0$ implies that non-topological solitons, periodic in time, are absent in the theory described by action (B.D).

\section{References}

[1] R.H. Hobart, On the instability of a class of unitary field models, Proc. Phys. Soc. 82 (1963) 201.

[2] G.H. Derrick, Comments on nonlinear wave equations as models for elementary particles, J. Math. Phys. 5 (1964) 1252.

[3] V.A. Rubakov, Classical theory of gauge fields, Princeton University press, Princeton 2002.

[4] N.S. Manton, Scaling identities for solitons beyond Derrick's theorem, J. Math. Phys. 50 (2009) $032901[0809.2891$ [hep-th]].

[5] V.G. Makhankov, On the existence of non-one-dimensional soliton like solutions for some field theories, Phys. Lett. A 61 (1977) 431.

[6] S. Dimopoulos, T. Eguchi, Virial theorems on instantons, Phys. Lett. B 66 (1977) 480.

N.V. Krasnikov, Implications of variational principle on instantons, Phys. Lett. B 72 (1978) 455.

S.R. Coleman, V. Glaser, A. Martin, Action minima among solutions to a class of euclidean scalar field equations, Commun. Math. Phys. 58 (1978) 211.

A. Actor, Properties of an instanton anti-instanton solution of a Yang-mills theory, Z. Phys. C 3 (1979) 353.

O.K. Pashaev, Classical solutions and "hidden" symmetry of Bose-Fermi system (In Russian), (1980) [Preprint: JINR-P2-80-16].

[7] W.A. Strauss, L. Vazquez, Existence of localized solutions for certain model field theories, J. Math. Phys. 22 (1981) 1005.

[8] G. Rosen, Nonexistence of localized periodic solutions to nonlinear field theories, Math. Phys. 7 (1966) 2071. 
[9] M.N. Smolyakov, Non-existence of nontopological solitons in some types of gauge field theories in Minkowski space, J. Math. Phys. 52 (2011) 102901; Erratum-ibid: 53 (2012) 019901 [1 012.0319 [math-ph] ].

[10] S. Deser, Absence of static solutions in source-free Yang-Mills theory, Phys. Lett. B 64 (1976) 463.

[11] H. Pagels, Absence of periodic solutions to scale invariant classical field theories, Phys. Lett. B 68 (1977) 466.

[12] S.R. Coleman, There are no classical glueballs, Commun. Math. Phys. 55 (1977) 113.

S.R. Coleman, L. Smarr, Are there geon analogs in sourceless gauge-field theories?, Commun. Math. Phys. 56 (1977) 1.

R. Weder, Absence of classical lumps, Commun. Math. Phys. 57 (1977) 161.

M. Magg, Some constraints on finite energy solutions in nonabelian gauge theories, J. Math. Phys. 19 (1978) 991.

R.T. Glassey, W.A. Strauss, Decay of classical Yang-mills fields, Commun. Math. Phys. 65 (1979) 1.

[13] G. Rosen, Exact solutions to the Yang-Mills field equations, J. Math. Phys. 13 (1972) 595.

[14] R.T. Glassey, W.A. Strauss, Decay of a Yang-mills field coupled to a scalar field, Commun. Math. Phys. 67 (1979) 51.

[15] G. Rosen, Charged particlelike solutions to nonlinear complex scalar field theories, J. Math. Phys. 9 (1968) 999.

[16] A.B. Adib, (Non)existence of static scalar field configurations in finite systems, [hep-th/0208168]. 